\title{
Trajectories of Early Adolescent Loneliness: Implications for Physical Health and Sleep
}

\author{
Alice M. Eccles $\mathbb{B}^{1} \cdot$ Pamela Qualter $^{2} \cdot$ Margarita Panayiotou $^{2} \cdot$ Ruth Hurley ${ }^{1} \cdot$ Michel Boivin $^{3} \cdot$ Richard E. Tremblay $^{4}$
}

Published online: 29 August 2020

(c) The Author(s) 2020

\begin{abstract}
The current study examines the relationship between prolonged loneliness, physical health, and sleep among young adolescents (10-13 years; $N=1214 ; 53 \%$ girls). Loneliness was measured at 10,12 and 13 years of age along with parentreported health and sleep outcomes. Using growth mixture modelling, 6 distinct trajectories were identified: 'low increasing to high loneliness' $(n=23,2 \%)$, 'high reducing loneliness' $(n=28,3 \%)$, 'medium stable loneliness' $(n=60,5 \%)$, 'medium reducing loneliness' $(n=185,15 \%)$, 'low increasing to medium loneliness' $(n=165,14 \%)$, and 'low stable loneliness' $(n=743,61 \%)$. Further analyses found non-significant differences between the loneliness trajectories and parent-report health and sleep outcomes including visits to health professionals, perceived general health, and sleep quality. The current study offers an important contribution to the literature on loneliness and health. Results show that the relationship may not be evident in early adolescence when parent reports of children's health are used. The current study highlights the importance of informant choice when reporting health. The implications of the findings for future empirical work are discussed.
\end{abstract}

Keywords Loneliness $\cdot$ Health $\cdot$ Adolescent $\cdot$ Longitudinal $\cdot$ Sleep

\section{Highlights}

- Six distinct trajectories of loneliness were identified in a large, representative sample.

- Novel examination of loneliness and parent-reported sleep and health outcomes.

- Highlights the importance of measurement and informant choice for future work.

Loneliness is a negative emotional state caused by a discrepancy between a person's desired and actual social relationships (Peplau and Perlman 1982). Loneliness is related, but distinct from social isolation (Smith and Victor 2019); it is characterised by dissatisfaction with current

Supplementary Information The online version of this article (https://doi.org/10.1007/s10826-020-01804-3) contains supplementary material, which is available to authorised users.

Alice M. Eccles

AEccles1@uclan.ac.uk

1 School of Psychology, University of Central Lancashire, Preston, Lancashire, UK

2 Manchester Institute of Education, University of Manchester, Oxford Road, Manchester, UK

3 Université Laval, ,Québec QC G1V 0A6, Canada

4 The University of Montreal, Québec, Canada relationships (Cacioppo and Cacioppo 2014) and a feeling of emotional or physical disconnection from others (Qualter et al. 2015). It involves uncomfortable feelings, including sadness, which people try to alleviate quickly by reconnecting with others. Among adults, those feelings contribute to reductions in well-being, which for those who experience loneliness frequently contributes to lower reports of quality of life, and poorer overall physical and mental health (Leigh-Hunt et al. 2017). Much of the research linking loneliness and poor health is focused on adults and there is limited examination of the impact of prolonged loneliness on physical health and sleep among adolescents. The current study addresses that gap in the literature, extending our understanding of distinct developmental trajectories of loneliness in adolescence and their impact on health.

Loneliness is a common experience throughout life, peaking when there are substantial changes to our social environments (Qualter et al. 2015). Loneliness is considered a normative reaction to those changing social circumstances 
and psychological challenges; people are motivated to connect with others, finding groups to which they can belong and make friends with people they can trust. There are two life periods-adolescence and old age-characterised by major changes to social environments, where increased social needs and expectations increase sensitivity to feelings of loneliness (Victor and Yang 2012). Loneliness has been examined extensively among those in old age, but there is limited work exploring loneliness among youth despite empirical literature showing the experience is common among adolescents (Qualter et al. 2015). Recent data from population surveys (Australian Loneliness Report 2018; CIGNA 2018; Office of National Statistics 2018), charity reports (Coop Foundation 2018; Griffin 2010), and academic research (BBC Loneliness Experiment 2018) shows that feelings of loneliness are common among adolescents and young adults. Given the evidence that loneliness is a particular issue for youth, there is a need to explore how it impacts their lives: is it the case that the same health effects of loneliness found among the elderly are also evident among youth.

Peer relationships become increasingly important during early adolescence (Rubin et al. 2006): concerns relating to social standing begin to emerge (Haller et al. 2014), interactions with peers' increase, and independence from parents occurs (Smetana et al. 2015); such increased focus on peers amplifies feelings of difference, increasing loneliness. Most adolescents navigate those normative feelings of loneliness, but empirical evidence suggests there are small groups of individuals who are at risk of prolonged feeling of loneliness during those crucial years (Harris et al. 2013; Jobe-Shields et al. 2011; Ladd and Ettekal 2013; Qualter et al. 2013; Schinka et al. 2013; Vanhalst et al. 2013). Overall, the findings suggest that the vast majority of youth experience consistently low or moderate levels of loneliness and only a small minority suffers from chronically high levels of loneliness. While there is a growing body of evidence to support the presence of distinct loneliness trajectories, there is a need for further research that examines loneliness over time and whether following certain trajectories of loneliness predicts important developmental outcomes, including physical health and sleep.

The relationship between loneliness and physical health has been well researched among adults (for reviews see HoltLunstad et al. 2015; Valtorta et al. 2016) with prolonged loneliness leading to increasing blood pressure (Hawkley et al. 2006), higher incidence of coronary heart disease (Thurston and Kubzansky 2009), and poorer perceived general health (Nummela et al. 2011; Segrin and Domschke 2011) and health complaints, such as migraines (Christiansen et al. 2016). Continued activation of physiological systems, including the hypothalamic-pituitary-adrenocortical (HPA) axis, contribute to inflammatory processes and are thought to be the leading mechanism by which loneliness is linked to illhealth (Hawkley and Cacioppo 2010).

Within young populations, most research has used concurrent data and shown that higher levels loneliness are associated with higher odds of reporting headaches and stomach aches (Stickley et al. 2016), and poorer selfreported health (Eccles et al. 2020; Lohre 2012). The crosssectional nature of those studies is a problem because such an approach fails to consider individual variation in the experience of loneliness over time. Given the growing body of research that highlights that it is prolonged loneliness that links to poor health, it is crucial to distinguish between those who are chronically lonely and others for whom loneliness is a transitory experience. Among older adolescent samples, prolonged loneliness predicted later selfreported health (Qualter et al. 2013; Harris et al. 2013), was a metabolic risk factors associated with cardiovascular disease (Goosby et al. 2013), and increased the number of GP visits (Qualter et al. 2013). Work is needed to explore how young adolescents following different trajectories of loneliness differentially experience health.

As well as explore different trajectories of loneliness and how those link to health, it is important to control for earlier health reports in any exploration of how loneliness predicts poor health: self-reported health is stable from early adolescence through to young adulthood (Fosse and Haas 2009), and without controlling for earlier poor health, any analysis that explores the prospective link between loneliness and health is not robust. Such robustness is missing from the extant empricial work. In addition, the growing body of evidence that exposure to poverty during early childhood is associated with a wide variety of adult health conditions irrespective of concurrent deprivation status (Braveman and Barclay 2009), means there is a need to control socioeconomic status (SES) and family affluence when examining the effects of loneliness on later health. Seguin et al. (2012) examined the trajectory of socioeconomic status, including family income, using the QLSCD data from baselines through to 10 years of age. The trend data suggests the prevalence of poverty during childhood declines over time with fewer children living with families with low income or receiving state support. Despite that increase in family affluence, Sequin highlights how early poverty, despite the increase in affluence later in childhood, has detrimental impacts upon subsequent health as illustrated by previous research (Braveman et al. 2011; Luo and White 2005; Poulton et al. 2002). Given findings that early childhood SES is associated with an elevated risk of mortality in adulthood, regardless of the individual's current SES status (Galobardes et al. 2008), it is important to account for early exposure to social inequality that might shape future barriers in life that impact health (Ferraro et al. 2016). 
As well as physical health, social stress is linked to disturbed sleep (Dahl and Lewin 2002), suggesting that loneliness may be counterproductive to a good night's sleep. Loneliness signals to an individual to change something about their social environment. As such, it leads to an increased vigilance to social cues and increased activation of the HPA axis in response to that social stress (Hawkley and Cacioppo 2010). Previous research supports that idea: loneliness is not associated with the overall sleep quantity, but it affects overall quality of the sleep among adults (Cacioppo et al. 2002; Kurina et al. 2011). Extant literature that has used prospective designs shows loneliness over time strongly predicts poor sleep quality among adults (Hawkley et al. 2010). Despite empirical work examining the relationship between loneliness and sleep among adults, there is a paucity of research examining that association among youth. In older adolescents, loneliness has been associated with subjective sleep outcomes such as quality and daytime dysfunction (Matthews et al. 2017), but not related to objective measures such as sleep duration and latency (Doane and Thurston 2014). Majeno et al. (2018) highlight the importance of loneliness and social stress, suggesting loneliness partially mediates the relationship between discrimination and sleep in adolescents.

In early adolescents, loneliness has been found to be associated with increased difficulties and experiences of disturbed sleep, but not will overall sleep quantity (Eccles et al. 2020). Whilst those previous studies support an association between loneliness and sleep, they all examine concurrent data. There is little research examining the relationship over time. In 8-11 year olds, Harris et al. (2013) showed that children experiencing a relatively high reducing loneliness reported greater sleep disturbance compared to children who followed a low, stable trajectory of loneliness. Such work with children suggests that loneliness may impact sleep, even when social reconnection occurs. There is a need to further investigate whether different trajectories of loneliness among youth relate to sleep outcomes. Such work will establish whether loneliness over timeis associated with poorer sleep quality consistently across different samples of youth.

Another important aspect to consider is the use of parent reports of their child's health, which is commonplace in the literature (Garbarski 2014). The use of proxy reports and the agreement with self-reported outcomes has been extensively researched and findings have been mixed (Smith and Goldman 2011; Todd and Goldman 2013). Nolan (2016) examined both parent and child report health utilising data collected in the Young Lives survey: moderate correlations between the two informants support convergent validity; parent reports also displayed a stronger association with physical health indicators such as height. There is evidence to support parental-proxy reports of health and their use warrants further attention.

The current study examined the presence of distinct developmental trajectories of loneliness in a sample of Canadian adolescents aged 10-13 years. First, we examined how loneliness changed over the course of late childhood and early adolescence for the whole sample. Second, we explored whether youth followed particular trajectories of loneliness, and whether those following particular trajectories of loneliness experienced poorer health and sleep problems. Our overall aim was to provide data on the prospective associations between loneliness, physical health, and sleep among young adolescents. We controlled for earlier health problems and early family income in our analyses to determine the uniqueness of loneliness in predicting health and sleep outcomes.

\section{Method}

The data used in the current study were collected as part of the on-going Québec Longitudinal Study of Child Development (QLSCD). The QLSCD is a large birth cohort study organised by the Direction Santé Québec of the Institut de la Statistique du Québec. The prospective study includes singleton infants born in the Canadian provinces of Québec between 1997 and 1998. The QLSCD study took baseline measurements from 2223 infants and mothers when infants were between the ages of 15 and 36 weeks (mean age of 5 months). The study continued to collect data, on an annual or biannual basis, and is still an active research project. The current study focused on data collected when the participants were ages 10,12, and 13 years (in the current study, referred to as Times 1, 2, and 3 respectively). The QLSCD received ethical approval from the ethics committee of the Faulty of Medicine, the University of Montreal and the Direction Sante Quebec, Institut de la Statistique du Quebec.

\section{Participants}

In the current study, only participants who completed the loneliness questions at Time 1 of the current study (age 10 years) and at least one other time point (age 12 or 13 years) were included in the study. A total of 1214 children $(53 \%$ female) met those criteria, with $13.45 \%$ missing data. Little's (1988) Missing Completely at Random (MCAR) test suggested data were not $\operatorname{MCAR}\left(\chi^{2}(2116)=2762.12, p<\right.$ 0.001 ), but following recommendations (Enders 2010; Muthen and Muthen 1998-2020), we computed missing data using FIML on the latent loneliness variables in the growth curve analysis. Missing data on the distal outcomes were imputed using multiple imputation (following 
guidelines provided by Asparouhov and Muthen 2010). Data imputation is typically less biased than other techniques such as list wise deletion (Schafer and Graham 2002).

\section{Procedure}

Data used in this study were collected from both the adolescents and their mothers. Youth completed the loneliness questions when they were ages 10,12, and 13 years respectively. At the same time points, mothers completed measures that asked about their child's sleep and physical health. Parental socio-economic status data were collected from the mother when the children were 17 months olds.

\section{Measures}

\section{Loneliness}

Children were asked to rate, using a 3-point likert scale $(1=$ never, $2=$ sometimes, and $3=$ always $)$ how often they had felt the following in the past 2 weeks: (1) I have no one to talk to, (2) I feel alone, and (3) I feel left out. Those three items are similar to those in the 3-item short form of the UCLA (Hughes et al. 2004). The three item scores were summed, creating an overall loneliness score with total scores ranging from 3 to 9 . Higher scores represented higher loneliness. The loneliness measure demonstrated reasonably good internal reliability at all three time points $(\mathrm{T} 1-\alpha=$ 0.67 ; $22-\alpha=0.68 ; \mathrm{T} 3-\alpha=0.73$ ).

\section{Physical health}

The current study includes variables relating to mothers' perceptions of their child's physical health. Perceived general health. Mothers' were asked to rate their child's current general health $(1[$ Excellent $]-5[$ Poor $])$, such that a higher score represented poorer general health. Health professional visits. Mothers indicated which of the following health professionals they had consulted in the past 12 months about their child's health: general practitioner, paediatrician, medical doctor and/or public health nurse. The number of health professionals the mother had consulted was summed. A higher score indicated that the child had visited a higher number of different health professionals. Antibiotic Use. Mothers indicated how many times their child had taken a course of antibiotics in the past 6 months. They used the following 6-point scale: $1=$ none, $2=$ once, $3=$ twice, $4=3$ times $; 5=4$ or more, $6=$ one or more long term antibiotics, and $7=$ continuous treatment. Infection rates. Mothers were asked which of the following infections their child had suffered from in the past three months: gastro-intestinal, ear, bronchitis/pneumonia, cold/ flu/laryngitis, and other infections. A total number of infections variable was created, with a higher score indicating a higher frequency of infections.

\section{Sleep}

For sleep behaviour, mothers were asked about the quality and quantity of their child's sleep. For sleep quality, mothers were asked whether their child was drowsy or sleepy during the day with a higher score representing poorer quality sleep $(1=$ Never to $4=$ Always $)$. For sleep quantity, mothers were asked to indicate how long in total their child slept during the night (on average); they provided answers in hours and minutes. Sleep quantity was calculated in half hour intervals.

\section{Family Income}

When children were 17 months old, mothers provided information on household income, household size, and type of family residence. That information was used to calculate income sufficiency using the Statistics Canada's definition of low income, which considers the number of people in the household and the family zone of residence. Family income was coded as sufficient (1) or insufficient (2).

\section{Data Analyses}

First, we examined the prevalence of loneliness among the current sample and explored bivariate correlations between all variables. Next, we explored the changes in loneliness at the group level for children in the QLSCD when they were ages 10-13 years using latent growth curve modelling (LGCM). LGCM enabled us to estimate the initial level of loneliness at baseline (intercept) and the degree of change in loneliness from baseline across the time of the study (slope). Factor loadings were fixed at $0,2,3$ to represent the time difference between measurements. The model fit was assessed using common fit indices: chi square index, Root Mean Square Error of Approximation (RMSEA: should be less than 0.07; Steiger 2007), and Comparative Fit Index (CFI: a value of equal or greater than 0.95 is indicative of good fit; Hu and Bentler 1999). Next, using latent class growth analysis (LCGA; Jung and Wickrama 2008), we explored whether there were sub groups of children that followed distinct trajectories of loneliness from ages 10-13 years, and whether that differential development predicted health and sleep outcomes controlling for earlier health and family income. In the first stage of LCGA, the basic model was tested, exploring only the trajectories of loneliness and without any control variables. The optimal number of groups following distinct trajectories of loneliness was determined by comparing K-Class and K-1 class model based on Bayesian Information Criterion (BIC), sample-size 
adjusted Bayesian Information Criterion (aBIC), and Akaike's Information Criteria (AIC). A lower BIC, aBIC, and AIC are indicative of a better fit (McLachlan and Peel, 2000). Class number was also evaluated using the LoMendell-Rubin likelihood ratio test (LMRT) and entropy. For LMRT, a significant $p$ value suggests the model was significantly improved by the additional class when compared to previous solution; for entropy, a value close to 1 indicates less classification error and therefore representing a better fit (Nagin and Odgers 2010). The optimal number of groups was also determined based on theoretical underpinnings and previous findings (Nagin and Odgers 2010) to ensure substantive meaning to the findings. In the second stage of the LCGA, the relations between the groups following distinct trajectories of loneliness and later health and sleep outcomes were examined using the $\mathrm{BCH}$ approach (Bolck et al. 2004; Bakk and Vermunt 2016) in Mplus V8. $\mathrm{BCH}$ estimated the mean differences in health and sleep between the different trajectory classes using Wald's chi square test (Asparouhov and Muthén 2014). In the second stage of the LCGA, we controlled for earlier reports of family income and baseline measures of health in the model. All models were run in Mplus V8 (Muthen and Muthen 1998-2020). We followed van de Schoot et al. (2017)'s recommendations for writing up the analysis.

\section{Results}

\section{Demographics}

Table 1 includes variable information for the 1214 participants whose data were analysed. Of those participants, $54 \%$ identified as Canadian, $15 \%$ were French, $21 \%$ identified as both; the remaining $10 \%$ were from other ethnicities. The majority of the sample came from homes that had been classified as having sufficient income to meet basic needs when the child was 17 months of age. Descriptive statistics, including correlations within and between variables, as well as additional analysis relating to SES stabilities, are outlined in Supplementary Tables S1, S2.

\section{Changes in Loneliness Over Time}

Results from LGCA showed a significant intercept $(\beta 0=$ $3.82, p<0.001)$ and a non-significant slope $(\beta 1=0.008$, $p=0.568)$, such that participants on average scored 3.82 on the loneliness scale at baseline, and stayed relatively stable over time $\left(\chi^{2}(3)=242.65, p<0.001, \mathrm{CFI}=0.98\right.$, RMSEA $=0.07, \mathrm{CI}=0.024-0.118)$. The results demonstrated a significant variance in the intercept $(0.36, p=0.014)$ and some variation for slope $(0.30, p=0.018)$, justifying the examination of different trajectories of loneliness.
Table 1 Early Family Income and Loneliness reports for all participants $(N=1214)$

$$
\% \text { of sample } N
$$

\begin{tabular}{|c|c|c|c|}
\hline \\
\hline \\
\hline & \multicolumn{3}{|c|}{$\begin{array}{l}\text { Early Family Income } \\
\text { Sufficient } \\
\text { Insufficient }\end{array}$} \\
\hline & \multicolumn{3}{|c|}{ Time point 1 Time point 2 Time point 3} \\
\hline Mean age in years ${ }^{\mathrm{a}}$ & 10.14 & 12.13 & 13.13 \\
\hline $\begin{array}{l}\text { Mean loneliness } \\
\text { (range is 3-9) }\end{array}$ & $3.84(1.42)$ & $3.79(1.41)$ & $3.89(1.65)$ \\
\hline
\end{tabular}

Mean loneliness scores based on sum score of $\mathrm{T} 1, \mathrm{~T} 2$, and $\mathrm{T} 3$ loneliness scores. Data on early family income was collected when the children were aged 17 months. Income sufficiency was defined using the Statistics Canada's definition of low income, whilst also considering the number of people in the household and the family zone of residence. The low-income cut-off used by Statistics Canada is an income level from which, on average, a person (or family) spends $20 \%$ more of their total income on food, shelter, and clothing than is spent by similar persons or families in similar locations (https://www 150.statcan.gc.ca/n1/pub/75f0002m/2012002/lico-sfr-eng.htm)

${ }^{a}$ Mean age calculated for those who provided data at the corresponding time points (T1: 1180; T2 = 1186; T3 = 1099)

Table 2 Model Fit Indices for 2 through 7 class solutions

\begin{tabular}{lrrrrrr}
\hline Class & AIC & BIC & Adj BIC & Entropy & $\begin{array}{l}\text { LRT } \\
p \text { value }\end{array}$ & $n$ \\
\hline 2 & $10,531.49$ & $10,577.40$ & $10,548.82$ & 0.871 & $<0.001$ & 204 \\
3 & $10,318.77$ & $10,379.99$ & $10,341.875$ & 0.856 & 0.037 & 130 \\
4 & $10,101.22$ & $10,177.74$ & $10,130.09$ & 0.907 & 0.050 & 58 \\
5 & 9908.07 & 9999.90 & 9942.73 & 0.924 & 0.002 & 40 \\
6 & 9770.46 & 9877.59 & 9810.89 & 0.931 & 0.001 & 23 \\
7 & 9681.43 & 9803.87 & 9727.63 & 0.939 & 0.061 & 17 \\
\hline
\end{tabular}

AIC Akaike's Information Criteria, BIC Bayesian Information Criterion, Adj BIC adjusted Bayesian Information Criterion, Entropy, LRT Lo-Mendell-Rubin likelihood ratio test Value, $N$ number of individuals in the smallest class

Using LCGA, two through to seven-class solutions for loneliness were estimated, with Table 2 outlining the fit indices. Considering the model fit indices, and the theoretical underpinnings and minimal class size recommendations (Class $k>1 \%$ of $N$; Jung and Wickrama 2008), a 6class solution fit the data best.

The six distinct loneliness trajectories are as follows: low increasing to high loneliness $(n=23,2 \%)$, high reducing loneliness $(n=28,3 \%)$, medium stable loneliness $(n=60$, $5 \%)$, medium reducing loneliness $(n=185,15 \%)$, low increasing to medium loneliness $(n=165,14 \%)$, and low stable loneliness ( $n=743,61 \%$; see Table 3 and Fig. 1$)$.

Children following a low increasing to high loneliness trajectory reported low levels of loneliness at 10 years old, but reported increasingly levels of loneliness across the three years resulting in a high level of loneliness at age 13 . 
Table 3 Loneliness mean scores at ages 10, 12 and 13 years for the 6class solution

\begin{tabular}{lrrrrr}
\hline & $n$ & $\begin{array}{l}\% \text { of } \\
N\end{array}$ & 10 years & 12 years & 13 years \\
\hline $\begin{array}{l}\text { Low Increasing } \\
\text { to Medium }\end{array}$ & 165 & 14 & 3.32 & 4.32 & 5.44 \\
Medium Reducing & 185 & 15 & 5.31 & 3.71 & 3.61 \\
High Reducing & 38 & 3 & 7.46 & 4.68 & 4.39 \\
Low Stable & 743 & 61 & 3.25 & 3.41 & 3.20 \\
Low Increasing to High & 23 & 2 & 3.77 & 5.76 & 8.05 \\
Medium Stable & 60 & 5 & 5.77 & 5.57 & 6.35 \\
\hline
\end{tabular}

The range of scores on the loneliness measure is 3 to 9, with 9 representing high reports of loneliness

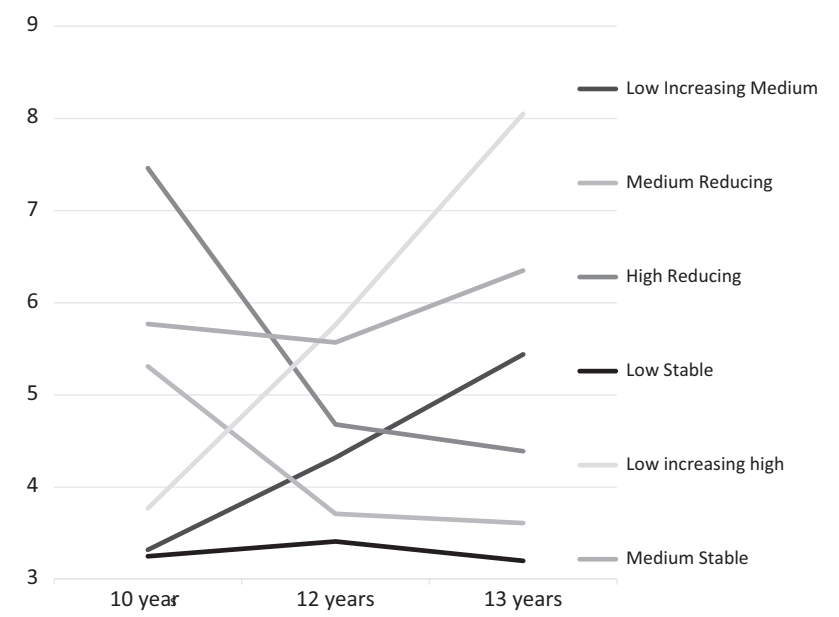

Fig. 1 Estimated mean trends for the six loneliness trajectory classes

Children following the high reducing loneliness trajectory reported relatively high levels of loneliness when they were 10 years of age, but that reduced over the next 3 years to average levels of loneliness. Children following the medium stable loneliness had relatively stable, but moderate, levels of loneliness over the three years. Those following the medium reducing trajectory reported average levels of loneliness at ten years of age, which reduced to lower than average over the next three years. Those children following the low increasing to medium loneliness trajectory reported low levels of loneliness at age 10 years and increased on loneliness steadily, to an average level of loneliness, over the next three years. The majority of children followed the low stable loneliness trajectory reporting low levels of loneliness from ages 10-13 years.

Using the $\mathrm{BCH}$ approach, the mean differences on adolescent health and sleep outcomes across the six different trajectories of loneliness were estimated. To control for Type 1 errors, a conservative adjusted alpha of $<0.001$ was used for all comparisons. In this step, we also controlled for the effects of earlier family income and health on later health and loneliness As shown in Table 4, we found no significant differences in physical health and sleep complaints between children who followed different trajectories of loneliness from 10-13 years.

\section{Discussion}

The current study examined the presence of groups of adolescents who followed distinct trajectories of loneliness from ages 10-13 years, with the aim of exploring the prospective link between prolonged loneliness and physical health and sleep outcomes among youth. We controlled for the impact of early family income, as a known predictor of poor health outcomes among adolescence and for earlier reports of poor health, exploring loneliness as a unique predictor of poor health and inadequate sleep. We found six distinct trajectories of loneliness-'Low Increasing to Medium', 'Medium Reducing', 'High Reducing', 'Low', 'Low Increasing to High' and 'Medium Stable'-but there was no support for the hypothesis that increasing or maintained loneliness was related to poorer health outcomes as reported by the mother. Given findings that loneliness is linked to poor health and sleep quality among adults, we discuss why we have different findings for youth in the current sample. We discuss why it might be the case that young adolescents who report higher loneliness, and particularly those in the current sample, might have mothers who do not report them as experiencing poor health and sleep outcomes.

Adolescents in the current sample followed one of six distinct trajectories of loneliness from 10 to 13 years of age: (1) 'Low Increasing to Medium', (2) 'Medium Reducing', (3) 'High Reducing', (4) 'Low Stable', (5) 'Low Increasing to High' and (6) 'Medium Stable'. The low stable group explained the loneliness experience for the majority of the participants (61\%), supporting previous research examining distinct trajectories of loneliness across adolescence (Harris et al. 2013; Jobe-Shields et al. 2011; Ladd and Ettekal 2013; Qualter et al. 2013; Schinka et al. 2013; Vanhalst et al. 2013). The current sample did not include a group of adolescents who followed a high stable trajectory of loneliness, a group found in some of the previous research (Ladd and Ettekal 2013; Qualter et al. 2013; Schinka et al. 2013), but there was evidence that some children followed a trajectory characterised by high reducing loneliness, and others followed a trajectory of low increasing to high loneliness. The most likely explanation for not finding a group of adolescents experiencing prolonged loneliness is the relatively low levels of loneliness reported by the current sample. Given other recent data suggesting that loneliness is a significant 
Table 4 Trajectory means and comparisons on physical health and sleep outcomes, controlling for early family income and health outcomes

\begin{tabular}{|c|c|c|c|c|c|c|}
\hline & Low Increasing to Medium & Medium Reducing & High Reducing & Low Stable & Low Increasing to High & Medium Stable \\
\hline Visits to Professionals & 1.57 & 1.06 & 1.60 & 1.30 & 2.28 & 2.65 \\
\hline Perceived Health & 0.72 & 0.95 & 0.76 & 0.68 & 0.90 & 0.26 \\
\hline Infection Rates & 5.51 & 4.98 & $3.28^{\mathrm{a}}$ & 5.10 & 5.04 & 4.75 \\
\hline Antibiotic Use & 1.06 & 1.06 & 0.78 & 0.89 & 1.32 & 1.14 \\
\hline Daytime Drowsiness & 0.97 & 0.73 & 0.61 & 0.66 & 1.34 & 0.79 \\
\hline Overall Sleep Quantity & 36.26 & 33.58 & 35.98 & 33.44 & 34.48 & 30.73 \\
\hline
\end{tabular}

No comparison demonstrated a significant factor as indicated by $p>0.05$

Mothers reported on all health complaints. All values reported for the outcomes are standardised residuals. Differences across profiles on T3/age 13 outcomes were obtained after controlling for earlier reports of respective physical health/sleep complaints (measured at T1/age 10) and early family income effects (data collected when the child was 17 months old)

Analysis were also completed without Earlier Family Income as a control and the results did not differ

${ }^{a}$ High reducing trajectory demonstrated lower rates of infection than the low stable $(p=0.010)$, medium reducing $(p=0.051)$ and low increasing $(p=0.004)$. However, to control for Type 1 errors, these comparisons failed to reach the Bonferroni adjusted $p<0.001$

issue for adolescents (for example, Office of National Statistics 2018), it becomes important to determine what is different about the current sample that makes these adolescents report lower levels of loneliness; given the current study includes a representative sample of youth we need further study to understand what might be different about the experiences of youth in Quebec or Canada that sets them apart from adolescents from other countries where reports of loneliness among youth are much higher. It is also note-worthy that the loneliness measure utilised in the current study is different from those in previous research, and may explain why higher levels of loneliness were not captured within the sample.

We did not find support for prospective relationships between loneliness and physical health and loneliness and sleep outcomes: adolescents in the current study following the different trajectories of loneliness did not significantly differ on the health and sleep outcomes. Previous research that examined different pathways of loneliness reported significant differences between distinct loneliness trajectories on health and sleep outcomes (Harris et al. 2013; Qualter et al. 2013), but our findings do not support that earlier work. The lack of relationship between self-reported loneliness and parent-reported health outcomes could be for a number of reasons. First, it may be that mothers do not accurately report the health and sleep of their children. Indeed, previous research suggests mother may underestimate sleep problems within children and adolescents (Fatima et al. 2016) with up to one third of potential sleep problems remaining unnoticed (Paavonen et al. 2000). Second, the negative cognitive bias and affect associated with loneliness could extenuate discrepancies between informants. Although adolescents are typically accurate at reporting their own physical health (Riley 2004), there is evidence to suggest adolescents are much less optimistic about their health than their parents as a result of a sensitivity or mental health problem the parents is not aware of
(Waters et al. 2003). It may be the case that self-reported health provides a 'window into the internal lives of the youth' (Johnson and Wang 2008); adolescents reporting loneliness are pessimistic and negative in their reflections, and that includes their health, supporting previous associations between youth loneliness, health and sleep (Qualter et al. 2013; Stickley et al. 2016; Matthews et al. 2017; Harris et al. 2013; Eccles et al. 2020). Further research should investigate the impact of different informants, including parents and health professionals, to help deepen our understanding about the true relationship between youth loneliness and health.

The current study offers an important contribution to the literature on loneliness and health. We showed that the relationship may not be evident in early adolescence when parent reports of children's health are used. However, the study is not without limitations. It is important to note the health measures were not from a validated scale, and the current research could be extended through the use of more objective measures of sleep, (e.g. sleep caps) and health biomarkers and cortisol). Extending research examining the relationship between youth loneliness and health and/or sleep through more objective measures, to mirror those used in adult literature, will help provide a deeper understanding behind the relationship as well as insight into the underlying mechanisms.

It is also important to recognise the overall levels of loneliness reported in the current sample are low. Given the reliability of the loneliness measure used it the current study, the current study highlights the importance of measurement choice. There are arguments for using short measures of loneliness with restricted response scales, but there are counterarguments that such measures do not truly capture the experience of youth loneliness (Eccles et al. 2020). However, Eccles et al. showed that associations between loneliness and health/sleep complaints were robust across the longer and shortened version of the UCLA when used with adolescents, 
suggesting that short measures such as that used in the current study should not represent a problem because they measure loneliness accurately. However, more research using both standard and shortened versions of loneliness measures are needed so the potential impact of how we measure loneliness can be examined. To help overcome that potential limitation, it is important that future work gathers prevalence and cross-national survey data relating to the presence, experience, and feeling of loneliness amongst young people, and the associated health outcomes.

With the limitations considered, the current study still offers a valuable contribution to the literature and has many merits. The study retains a large sample size throughout the analyses, and utilises a robust statistical method that considered the health differences between groups following different trajectories of loneliness, allowing for full analysis in one statistical programme (Bakk and Vermunt 2016). The longitudinal nature of the study is certainly a strength, enabling the examination of the relationship between loneliness and health outcomes over a prolonged period of time; the control of early family income and health also ensured robust control of important related constructs. The current study examined the prospective association between loneliness, physical health, and sleep, and we provided a robust design by controlling for early reports of family income and child health that have been shown to impact later health outcomes. Contrary to research with adults, we did not find a relationship between increasing loneliness and poor health and sleep quality. That was, to some extent, a reflection of the fact that young adolescents in the current representative sample of Canadian youth did not report high levels of loneliness. Future research will want to (1) explore feelings of loneliness in different groups to establish whether high, stable loneliness over time impacts health negatively, and (2) gather prospective health data from multiple sources, including via objective measurement, to explore whether the relationship between loneliness and poor health is primarily driven by self-perceptions.

Acknowledgements This study uses data from the Quebec Longitudinal Study of Child Development (QLSCD). Data collection for the QLSCD was made possible through funding from the ministère de la Santé et des Services Sociaux (MSSS) (Ministry of Health and Social Services), the Lucie and André Chagnon Foundation, the ministère de la Famille (Ministry of Family), and the Institut de la statistique du Québec (the Institut).

Author Contributions A.E. conceived the study, performed statistical analyses, interpreted the findings, drafted the manuscript and made revisions to the manuscript based on reviewer feedback; P.Q. conceived the study, helped to perform statistical analyses, interpret the findings, and contributed to the draft manuscript; M.P. and R.H. helped to perform statistical analyses and interpret the findings, and contributed to the draft manuscript; M.B. and R.E.T. participated in the study design, and edited the manuscript; M.B. and R.E.T. coordinated the original data collection. All authors read and approved the final manuscript.

Data Sharing and Declaration The dataset analysed during the current study is not publicly available; It is available from Institut de la statistique du Québec (Information and Documentation Centre, Institut de la statistique du Québec, 200, chemin Sainte-Foy, 3e étage Québec (Québec, G1R 5T4).

\section{Compliance with Ethical Standards}

Conflict of Interest The authors declare that they have no conflict of interest.

Ethical Approval All procedures performed in studies involving human participants were in accordance with the ethical standards of the institutional and/or national research committee (include name of committee + reference number) and with the '1964 Helsinki declaration and its later amendments or comparable ethical standards.'

Informed Consent Informed consent was obtained from all individual participants included in the study.

Publisher's note Springer Nature remains neutral with regard to jurisdictional claims in published maps and institutional affiliations.

Open Access This article is licensed under a Creative Commons Attribution 4.0 International License, which permits use, sharing, adaptation, distribution and reproduction in any medium or format, as long as you give appropriate credit to the original author(s) and the source, provide a link to the Creative Commons license, and indicate if changes were made. The images or other third party material in this article are included in the article's Creative Commons license, unless indicated otherwise in a credit line to the material. If material is not included in the article's Creative Commons license and your intended use is not permitted by statutory regulation or exceeds the permitted use, you will need to obtain permission directly from the copyright holder. To view a copy of this license, visit http://creativecommons. org/licenses/by/4.0/.

\section{References}

Asparouhov, T., \& Muthen, B. (2010). Multiple Imputation with Mplus. https://www.statmodel.com/download/Imputations7.pdf. Accessed Aug 2020.

Asparouhov, T., \& Muthén, B. (2014). Auxiliary variables in mixture modeling: using the $\mathrm{BCH}$ method Mplus to estimate a distal outcome model and an arbitrary secondary model. Mplus Web Notes: No. 21. www.statmodel.com.

Australian Loneliness Report (2018). A survey exploring the loneliness levels of Australians and the impact on their health and wellbeing. https://psychweek.org.au/wp/wp-content/uploads/ 2018/11/Psychology-Week-2018-Australian-Loneliness-Report. pdf. Accessed 6 Feb 2019.

Bakk, Z., \& Vermunt, J. K. (2016). Robustness of stepwise latent class modeling with continuous distal outcomes. Structural Equation Modeling, 23, 20-31. https://doi.org/10.1080/ 10705511.2014.955104.

BBC Loneliness Experiment (2018). Who feels lonely? The results of the world's largest loneliness study. https://www.bbc.co.uk/ programmes/articles/2yzhfv4DvqVp5nZyxBD8G23/who-feelslonely-the-results-of-the-world-s-largest-loneliness-study. Accessed 6 Feb 2019. 
Bolck, A., Croon, M., \& Hagenaars, J. (2004). Estimating latent structure models with categorical variables: One-step versus three-step estimators. Political Analysis, 12(1), 3-27. https://doi. org/10.1093/pan/mph001.

Braveman, P., \& Barclay, C. (2009). Health disparities beginning in childhood: a life-course perspective. Pediatrics, 124, 163-175. https://doi.org/10.1542/peds.2009-1100D.

Braveman, P., Egerters, S., \& Williams, D. R. (2011). The social determinants of health: coming of age. Annual Review of Public Health, 32(1), 381-398. https://doi.org/10.1146/annurev-publhea lth-031210-101218.

Cacioppo, J. T., \& Cacioppo, S. (2014). Social relationships and health: the toxic effects of perceived social isolation. Social and Personality Compass, 8(2), 58-72. https://doi.org/10.1111/spc3.12087.

Cacioppo, J. T., Hawkley, L. C., Bernston, G. G., Ernst, J. M., Gibbs, A. C., Stickgold, R., \& Hobson, J. A. (2002). Do lonely days invade the nights? Potential social modulation of sleep efficiency. Psychological Science, 13(4), 384-387.

Christiansen, J., Larsen, F. B., \& Lasgaard, M. (2016). Do stress, health behaviour, and sleep mediate the association between loneliness and adverse health condition among older people? Social Science and Medicine, 152, 80-86. https://doi.org/10. 1016/j.socscimed.2016.01.020.

CIGNA (2018). US Loneliness Index. https://www.multivu.com/players/ English/8294451-cigna-us-loneliness-survey/docs/IndexReport_ 1524069371598-173525450.pdf. Accessed 6 Feb 2019.

Coop Foundation (2018). All our emotions are important: Breaking the silence about youth loneliness. https://assets.ctfassets.net/eua 7b5q6or8q/1pcX29wfVuOoouCGWkyqoc/4cce48d22c4a 9ce7d7ef69318f90a91a/Co-op_foundation_youth_loneliness_ report.pdf. Accessed 6 Feb 2019.

Dahl, R. E., \& Lewin, D. S. (2002). Pathways to adolescent health sleep regulation and behaviour. Journal of Adolescent Health, 31 (6), 175-184. https://doi.org/10.1016/S1054-139X(02)00506-2.

Doane, L. D., \& Thurston, E. C. (2014). Associations among sleep, daily experiences, and loneliness in adolescence: evidence of moderating and bidirectional pathways. Journal of Adolescence, 37(2), 145-154. https://doi.org/10.1016/j.adolescence.2013.11.009.

Eccles, A. M., Qualter, P., Madsen, K. R., \& Holstein, B. E. (2020). Loneliness in the lives of Danish adolescents: Associations with health and sleep. Scandinavian journal of public health, 1403494819865429. Advance online publication. https://doi.org/ $10.1177 / 1403494819865429$

Enders, C.K. (2010). Applied missing data analysis. New York, US: Guilford Press.

Fatima, Y., Doi, S. A., O'Callaghan, M., Williams, G., Najman, J. M., \& Mamun, A. A. (2016). Parent and adolescent reports in assessing adolescent sleep problems: results from a large population study. Acta Paediatrica, 105, e433-e439. https://doi.org/10.1111/apa.13404.

Ferraro, F. F., Schafer, M. H., \& Wilkinson, L. R. (2016). Childhood disadvantage and health problems in middle and later life. Early imprints on physical health?. American Sociological Review, 81 (1), 107-133. https://doi.org/10.1177/0003122415619617.

Fosse, N. E., \& Haas, S. A. (2009). Validity and stability of selfreported health among adolescents in a longitudinal, nationally representative survey. Pediatrics, 123(3), e496-e501. https://doi. org/10.1542/peds.2008-1552.

Galobardes, B., Lynch, J. W., \& Smith, G. D. (2008). Is the association between childhood socioeconomic circumstances and causespecific mortality established? Update of a systematic review. Journal of Epidemiology and Community Health, 62(5), 387-380. https://doi.org/10.1136/jech.2007.065508.

Garbarski, D. (2014). Comparing self and maternal reports of adolescents' general health status: do self and proxy reports differ in their relationships with covariates? Quality of Life Research, 23, 1953-1965. https://doi.org/10.1007/s11136-014-0649-0.
Goosby, B. J., Bellatorre, A., Walsemann, K. M., \& Cheadle, J. E. (2013). Adolescent loneliness and health in early adulthood. Sociological Inquiry, 83(4), 1-22. https://doi.org/10.1111/soin.12018.

Griffin, J. (2010). The lonely society. London: Mental Health Foundation.

Haller, S. P., Cohen, Kadosh, K., \& Lau, J. Y. (2014). A developmental angle tfo understanding the mechanisms of biased cognitions in social anxiety. Frontiers in Human Neuroscience, 7, 846. https://doi.org/10.3389/fnhum.2013.00846.

Harris, R. A., Qualter, P., \& Robinson, S. J. (2013). Loneliness trajectories from middle childhood to pre adolescence: impact of perceived health and sleep disturbance. Journal of Adolescence, 36, 1295-1304. https://doi.org/10.1016/j.adolescence.2012.12.009.

Hawkley, L. C., \& Cacioppo, J. T. (2010). Loneliness matters: A theoretical and empirical review of consequences and mechanisms. Annals of Behavioral Medicine, 40(2), 218-227. https://doi. org/10.1007/s12160-010-9210-8.

Hawkley, L. C., Masi, C. M., Berry, J. D., \& Cacioppo, J. T. (2006). Loneliness is a unique predictor of age related difference in systolic blood pressure. Psychology \& Aging, 21(1), 152-164. https://doi.org/10.1037/0882-7974.21.1.152.

Holt-Lunstad, J., Smith, T. B., Baker, M., Harris, T., \& Stephenson, D. (2015). Loneliness and social isolation as risk factors for mortality: a meta-analytic review. Perspectives on Psychological Science, 10 (2), 227-237. https://doi.org/10.1177/1745691614568352.

Hu, L., \& Bentler, P. M. (1999). Cut-off criteria for fit indexes in covariance structure analysis: conventional criteria versus new alternatives. Structural Equation Modeling: A Multidisciplinary Journal, 6(1), 1-55. https://doi.org/10.1080/10705519909540118.

Hughes, M. E., Waite, L. J., Hawley, L. C., \& Cacioppo, J. T. (2004). A short scale for measuring loneliness in large surveys: Results from two population based studies. Research on Aging, 26(6), 655-672.

Jobe-Shields, L., Cohen, R., \& Parra, G.R. (2011) Patterns of change in children's loneliness: trajectories from third through fifth grades, Merrill-Palmer Quarterly, 57(1), 25-47. https://digita lcommons.wayne.edu/mpq/vol57/iss $1 / 4$.

Johnson, S.B. \& Wang, C. (2008). Why do adolescents say they are less healthy than their parents think they are? The importance of mental health varies by social class in a nationally representative sample. Pediatrics, 121(e307). https://doi.org/10.1542/peds.2007-0881.

Jung, T., \& Wickrama, K. A. S. (2008). An Introduction to Latent Class Growth Analysis and Growth Mixture Modelling. Social and Personality Psychology Compass, 2(1), 302-317. https://doi. org/10.1111/j.1751-9004.2007.00054.x.

Kurina, L., Knutson, M., Hawkley, K. L., Cacioppo, L. C., Lauderdale, J. T., \& Ober, C. (2011). Loneliness is associated with sleep fragmentation in a communal society. Sleep, 34(11), 1519-1526. https://doi.org/10.5665/sleep.1390.

Ladd, G. W., \& Ettekal, L. (2013). Peer-related loneliness across early to late adolescence: normative trends, intra-individual trajectories, and links with depressive symptoms. Journal of Adolescence, 36(6), 1269-1282. https://doi.org/10.1016/j.adolescence.2013.05.004.

Leigh-Hunt., N., Bagguley, D., Bash, K., Turner, V., Turnbull, S., Valtorta, N., \& Caan, W. (2017). An overview of systematic reviews on the public health consequences of social isolation and loneliness. Public Health, 152, 157-171. https://doi.org/10.1016/j.puhe.2017.07.035.

Little, R. J. A. (1988). A test of missing completely at random for multivariate data with missing values. Journal of the American Statistical Association, 83(404), 1198-1202.

Lohre, A. (2012). The impact of loneliness on self-rated health symptoms among victimized school children. Child and Adolescent Psychiatry and Mental Health, 6(1), 20. https://doi.org/10.1186/1753-2000-6-20.

Luo, Y., \& White, L. J. (2005). The Impact of childhood and adult SES on physical, mental and cognitive well-being in later life. Journal of Gerontology Series B, Psychological Sciences and Social Sciences, 60(2), 93-101. 
McLachlan, G. J., \& Peel, D. (2000). Finite mixture models. New York: Wiley.

Majeno, A., Tsai, K. M., Huynh, V. W., McCreath, H., \& Fuligni, A. J. (2018). Discrimination and sleep difficulties during adolescence: the mediating roles of loneliness and perceived stress. Journal of Youth Adolescence, 47(1), 135-147. https://doi.org/10. 1007/s10964-017-0755-8.

Matthews, T., Danese, A., Gregory, A.M., Caspi, A., Moffitt, T.E., \& Arsenault, L. (2017). Sleeping with one eye open: loneliness and sleep quality in young adults. Psychological Medicine, 1-10. https://doi.org/10.1017/S0033291717000629.

Muthen, B., \& Muthen, L. (1998-2020). Mplus User's Guide. Los Angeles: Muthen and Muthen.

Nagin, D. S., \& Odgers, C. L. (2010). Group based trajectory modelling in clinical research. Annual Review of Clinical Psychology, 6, 109-138. https://doi.org/10.1146/annurev.clinpsy.121208.131413.

Nolan, L. B. (2016). An exploration of proxy and self-reported adolescent health in low resource settings. Survey Research Methods, 10(2), 65-83. https://doi.org/10.18148/srm/2016.v10i2.6711.

Nummela, O., Seppanen, M., \& Uutela, A. (2011). The effect of loneliness and change in loneliness on self-rated health: a longitudinal study among ageing people. Archives of Gerontology and Geriatrics, 53 (2), 163-167. https://doi.org/10.1016/j.archger.2010.10.023.

Office of National Statistics (2018). Loneliness-What characteristics and circumstances are associated with feeling lonely? https://www.ons.gov.uk/peoplepopulationandcommunity/w ellbeing/articles/lonelinesswhatcharacteristicsandcircumstancesa reassociatedwithfeelinglonely/2018-04-10. Accessed 6 Feb 2019.

Paavonen, E. J., Aronen, E. T., Moilanen, I., Piha, J., Räsänen, E., Tamminen, T., \& Almqvist, F. (2000). Sleep problems of schoolaged children: a complementary view. Acta Paediatrica, 89(2), 223-228. https://doi.org/10.1080/080352500750028870.

Peplau, L., \& Perlman, D. (1982). Perspectives on loneliness. In L. Peplau \& D. Perlman (Eds), Loneliness: a sourcebook of current theory, research and therapy (pp. 1-18). New York, NY: John Wiley and Sons.

Poulton, R., Caspi, A., Milne, B. J., Thomson, W. M., Taylor, A., Sears, M. R., \& Moffitt, T. E. (2002). Associations between children's experiences of socioeconomic disadvantage and adult health: a life-course study. Lancet, 23, 1640-1645. https://doi. org/10.1016/S0140-6736(02)11602-3.

Qualter, P., Brown, S. L., Rotenberg, K. J., Vanhalst, J., Harris, R. A., Goossens, L., Bangee, M., \& Munn, P. (2013). Trajectories of loneliness during childhood and adolescence: predictors and health outcomes. Journal of Adolescence, 36(6), 1283-1293. https://doi.org/10.1016/j.adolescence.2013.01.005.

Qualter, P., Vanhalst, J., Harris, R., Van Roekel, E., Lodder, G. et al. (2015). Loneliness across the life span. Perspectives on Psychological Science, 10(2), 250-264. https://doi.org/10.1177/ 1745691615568999.

Riley, A. W. (2004). Evidence that school age children can self-report their health. Ambulatory Paediatrics, 4(4), 371-376. https://doi. org/10.1367/A03-178R.1.

Rubin, K. H., Bukowski, W. M., \& Parker, J. G. (2006). Peer interactions, relationships, and groups. In N. Eisenberg, W. Damon, \& R. M. Lerner (Eds), Handbook of child psychology: social, emotional, and personality development. (pp. 571-645). NJ, US: John Wiley \& Sons Inc.

Schafer, J. L., \& Graham, J. W. (2002). Missing data: our view of the state of the art. Psychological Methods, 7(2), 147-177. https:// doi.org/10.1037//1082-989X.7.2.147.

Schinka, K. C., van Dulmen, M. H., Mata, A. D., Bossarte, R., \& Swahn, M. (2013). Psychosocial predictors and outcomes of loneliness trajectories form childhood to early adolescence. Journal of Adolescents, 36(6), 1251-160. https://doi.org/10.1016/ j.adolescence.2013.08.002.

Seguin, L., Nikiema, B., \& Gauvin, L. et al. (2012). Tracking Exposure to Child Poverty During the First 10 Years of Life in a Quebec Birth Cohort. Journal of Public Health, 103, e270-e276. https://doi.org/10.1007/BF03404234.

Segrin, C., \& Domschke, T. (2011). Social support, loneliness, recuperative processes, and their direct and indirect effects of health. Health Community, 269(3), 221-232. https://doi.org/10. 1080/10410236.2010.546771.

Smetana, J. G., Robinson, J., \& Rote, W. M. (2015). Socialization in adolescence. In J. E. Grusec \& P. D. Hastings (Eds), Handbook of socialization: theory and research (pp. 60-84). New York, US: Guilford Press.

Smith, K. V., \& Goldman, N. (2011). Measuring health status: self-, interviewer, and physician reports of overall health. Journal of Aging and Health, 23(2), 242-266. https://doi.org/10.1177/ 0898264310383421.

Smith, K., \& Victor, C. (2019). Typologies of loneliness, living alone and social isolation and their associations with physical and mental health. Ageing and Society, 39(8), 1709-1730. https://doi. org/10.1017/S0144686X18000132.

Steiger, J. H. (2007). Understanding the limitation of global fit assessment in structural equation modelling. Personality and Individual Differences, 42(5), 893-898. https://doi.org/10.1016/j. paid.2006.09.017.

Stickley, A., Koyanagi, A., Koposov, R., Blatný, M., Hrdlička, M., Schwab-Stone, M., \& Ruchkin, V. (2016). Loneliness and its association with psychological and somatic health problems among Czech, Russian and U.S. adolescents. BMC Psychiatry, 16, 128. https://doi.org/10.1186/s12888-016-0829-2.

Thurston, R. C., \& Kubzansky, L. D. (2009). Women, loneliness, and incident coronary heart disease. Psychosomatic Medicine, 71(8), 836-842. https://doi.org/10.1097/PSY.0b013e3181b40efc.

Todd, M. A., \& Goldman, N. (2013). Do interviewer and physician health ratings predict mortality? A comparison with self-rated health. Epidemiology, 24(6), 913-920. https://doi.org/10.1097/ EDE.0b013e3182a713a8.

Valtorta, N. K., Kanaan, M., Gilbody, S., Ronzi, S., \& Hanratty, B. (2016). Loneliness and social isolation as risk factors for coronary heart disease and stroke: systematic review and meta-analysis of longitudinal observational studies. Heart, 102, 1009-1016. https://doi.org/10.1136/heartjnl-2015-308790.

van de Schoot, R., Sijbrandij, M., Winter, S. D., Depaoli, S., \& Vermunt, J. K. (2017). The GRoLTS-checklist: guidelines for reporting on latent trajectory studies. Structural Equation Modeling: A multidisciplinary Journal, 24(3), 451-467. https://doi. org/10.1080/10705511.2016.1247646.

Vanhalst, J., Goossens, L., Luyckx, K., Scholte, R. H. J., \& Engels, R. C. M. E. (2013). The development of loneliness from mid- to late adolescence: trajectory classes, personality traits, and psychosocial functioning. Journal of Adolescence, 36(6), 1305-1312. https://doi.org/10.1016/j.adolescence.2012.04.002.

Victor, C. R., \& Yang, K. (2012). The prevalence of loneliness among adults: a case study of the United Kingdom.The Journal of Psychology, 146(1-2), 85-04. https://doi.org/10.1080/00223980.2011. 613875.

Waters, E., Stewart-Brown, S., \& Fitzpatrick, R. (2003). Agreement between adolescent self-report and parent reports of health and wellbeing: results of an epidemiological study. Child Care and Health Development, 29(6), 501-509. https://doi.org/10.1046/j. 1365-2214.2003.00370.x. 